\title{
Performance Comparison of Wavelet and Multiwavelet Denoising Methods for an Electrocardiogram Signal
}

\author{
Balambigai Subramanian, ${ }^{1}$ Asokan Ramasamy, ${ }^{2}$ and Kamalakannan Rangasamy ${ }^{1}$ \\ ${ }^{1}$ Department of Electronics and Communication Engineering, Kongu Engineering College, Perundurai, Erode District, \\ Tamil Nadu 638052, India \\ ${ }^{2}$ Kongunadu College of Engineering and Technology, Thottiyam, Trichy District, Tamil Nadu 621215, India
}

Correspondence should be addressed to Balambigai Subramanian; bharathian007@yahoo.com

Received 22 January 2014; Revised 14 April 2014; Accepted 17 April 2014; Published 11 May 2014

Academic Editor: Feng Gao

Copyright (c) 2014 Balambigai Subramanian et al. This is an open access article distributed under the Creative Commons Attribution License, which permits unrestricted use, distribution, and reproduction in any medium, provided the original work is properly cited.

\begin{abstract}
The increase in the occurrence of cardiovascular diseases in the world has made electrocardiogram an important tool to diagnose the various arrhythmias of the heart. But the recorded electrocardiogram often contains artefacts like power line noise, baseline noise, and muscle artefacts. Hence denoising of electrocardiogram signals is very important for accurate diagnosis of heart diseases. The properties of wavelets and multiwavelets have better denoising capability compared to conventional filtering techniques. The electrocardiogram signals have been taken from the MIT-BIH arrhythmia database. The simulation results prove that there is a $29.7 \%$ increase in the performance of multiwavelets over the performance of wavelets in terms of signal to noise ratio (SNR).
\end{abstract}

\section{Introduction}

In modern medicine, there are many methods to diagnose heart disease such as electrocardiogram (ECG), ultrasound, magnetic resonance imaging (MRI), and computer tomography (CT). Among these methods, diagnosis using electrocardiogram has the advantages of convenience and low cost so that it can be used in a wide area. However, certain arrhythmia (a fast, slow, or irregular heartbeat) which can cause abnormal symptoms may occur only sporadically or may occur only under certain conditions such as stress. Arrhythmia of this type is difficult to obtain on an electrocardiogram tracing that runs only for a few minutes. The electrocardiogram is the record of variation of bioelectric potential with respect to time as the human heart beats. Due to its ease of use and noninvasiveness, electrocardiogram plays an important role in patient monitoring and diagnosis.

The change in solar activity including electrocardiographic data with variations in galactic cosmic rays, geomagnetic activity, and atmospheric pressure suggests the possibility of links among these physical environmental variations and health risks, such as myocardial infarctions and ischemic strokes. An increase in the incidence of myocardial infarction in association with magnetic storms has been reported by Cornélissen et al. [1].

Magnetic storms are found to decrease heart rate variability (HRV) indicating a possible mechanism since a reduced HRV is an important factor for coronary artery disease and myocardial infarction. An increase of 5\% in mortality during years of maximal solar activity is found when compared with years of minimal solar activity. These chronodiagnostics are particularly important for those venturing into regions away from hospitals.

Goudarzi et al. [2] made an effort to find the optimum multiwavelet for compression of ECG signals to be used along with SPIHT codec. This work examined different multiwavelets on 24 sets of ECG data with entirely different characteristics selected from MIT-BIH database and assessed the functionality of the different multiwavelets in compressing electrocardiogram signals and their simulation results showed the cardinal balanced multiwavelet (cardbal2) by the means of identity (Id) prefiltering method to be the best effective transformation and the most efficient multiwavelet was applied for SPIHT coding algorithm on the transformed signal by this multiwavelet. 
Kania et al. [3] studied the application of wavelet denoising in noise reduction of multichannel high resolution ECG signals. The influence of the selection of wavelet function and the choice of decomposition level on efficiency of denoising process was considered and whole procedures of noise reduction were implemented in MATLAB environment using the fast wavelet transform. The denoising method was found to be advantageous since noise level was decreased in ECG signals, in which noise reduction by averaging had limited application, that is, in case of arrhythmia.

Helenprabha and Natarajan [4] proposed a technique used for measuring electrical signals generated by foetal heart as measured from multichannel potential recordings on the mother's body surface. They proposed a new class of adaptive filter that combines the attractive properties of finite impulse response (FIR) filter with infinite impulse response (IIR) filter. The maternal ECG and foetal signals were simulated using MATLAB. The gamma filter design was implemented in FPGA Spartan 2E which was programmed using VHDL. Their results have solved the complex situations more reliably than normal adaptive methods used earlier for recovering foetal signals.

Chang et al. [5] proposed measures to make the optimal filter design under different constraints possible for ECG signal processing. Experiments have been conducted by them with artificially and practically corrupted ECG signals for PLI adaptive filtering technique. The assessments included the convergence time, the frequency tracking efficiency, the execution time, and the relative statistics in time and frequency domain. The results demonstrated that there is no universal optimum approach for this application thus far.

Alfaouri and Daqrouq [6] performed wavelet transform thresholding technique for ECG signal denoising. They decomposed the signal into five levels of wavelet transform using the Daubechies wavelet (db4) and determined a threshold through a loop to find the value where minimum error was achieved between the detailed coefficients of threshold noisy signal and the original signal. The threshold value was accomplished experimentally after using a loop of calculating a minimum error between the denoised wavelet subsignals and the original free of noise subsignals. The experimental application of the threshold result was better than Donoho's threshold particularly in ECG signal denoising.

Zhidong and Chan [7] proposed a novel method for the removal of power line frequency from ECG signals based on empirical mode decomposition (EMD) and adaptive filter. A data-driven adaptive technique called EMD was used to decompose ECG signal into a series of intrinsic mode functions (IMFs). The adaptive power line cancellation filter was designed to remove the power line interference, the reference signal of which was produced by selective reconstruction of IMFs. Clinic ECG signals were used to evaluate the performance of the filter. Results indicated that the power line interference of ECG was removed effectively by the new method.

Kaur and Singh [8] proposed a combination method for power line interference reduction in ECG. The methods were moving average method and using the IIR notch characteristics. Their results showed reduction in the power line noise in the ECG signal using the proposed filter that has fewer coefficients and hence lesser computation time for real time processing.

Haque et al. [9] used wavelet method to detect the small variations of ECG features. They simulated standard ECG signals as well as the simulated noise corrupted signal using FFT and wavelet for proper feature extraction. They found wavelet to be superior to the conventional FFT method in finding the small abnormalities in electrocardiogram signals.

Tan and Lei [10] used wavelet transform to filter out noise interferences of electrocardiogram signals for the filtering of the myoelectric interference, the power frequency interference, and the baseline drift. Firstly Coif4 wavelet was adapted to decompose electrocardiogram signals containing noises. Secondly, the soft and hard threshold value quantified high-frequency coefficients of every scale and finally the electrocardiogram were reconstructed using high-frequency coefficients of every scale which were quantified by the threshold value. Experiments showed that wavelet transform had good real time filtering effect and it had more advantages than traditional methods.

\section{Materials and Methods}

2.1. Wavelet Method. A wavelet is simply a small wave which has energy concentrated in time. It is compactly supported and has finite energy function. It can satisfy admissibility condition and could be amendable for multiresolution analysis. The wavelet transform is a mathematical tool for decomposing a signal into a set of orthogonal waveforms localized both in time and frequency domains. The wavelet transform is a suitable tool to analyse the electrocardiogram signal, which is characterized by a cyclic occurrence of patterns with different frequency content $(P$ wave, QRS complex, and $T$ wave). It is a decomposition of the signal as a combination of a set of basic functions, obtained by means of dilation $(a)$ and translation $(b)$ of a single prototype wavelet; there are several wavelet functions (mother wavelet with different properties) like the Morlet or Mexican Hat wavelets or complex frequency $B$ spline wavelets that are used in study.

Wavelet analysis is done by the breaking up of a signal into a shifted and scaled version of the original wavelet. A continuous wavelet transform can be defined as the sum of overall time of the signal multiplied by a scaled and shifted version of the wavelet function. The greater the scale factor " $a$ " is, the wider the basis function is and, consequently, the corresponding coefficient gives information about lower frequency components of the signal and vice versa.

The wavelet transform is designed to address the problem of nonstationary signals such as electrocardiogram signals. It involves representing a time function in terms of simple, fixed building blocks, and termed wavelets. The next step is the selection of number of decomposition levels of signal $x_{i}(t)$; see Figure 1 . First decomposition level is obtained by using two complementary high- and low-pass filters and then half of the samples are eliminated. The filters cut frequency is equal to half of the bandwidth of analysed signal. 


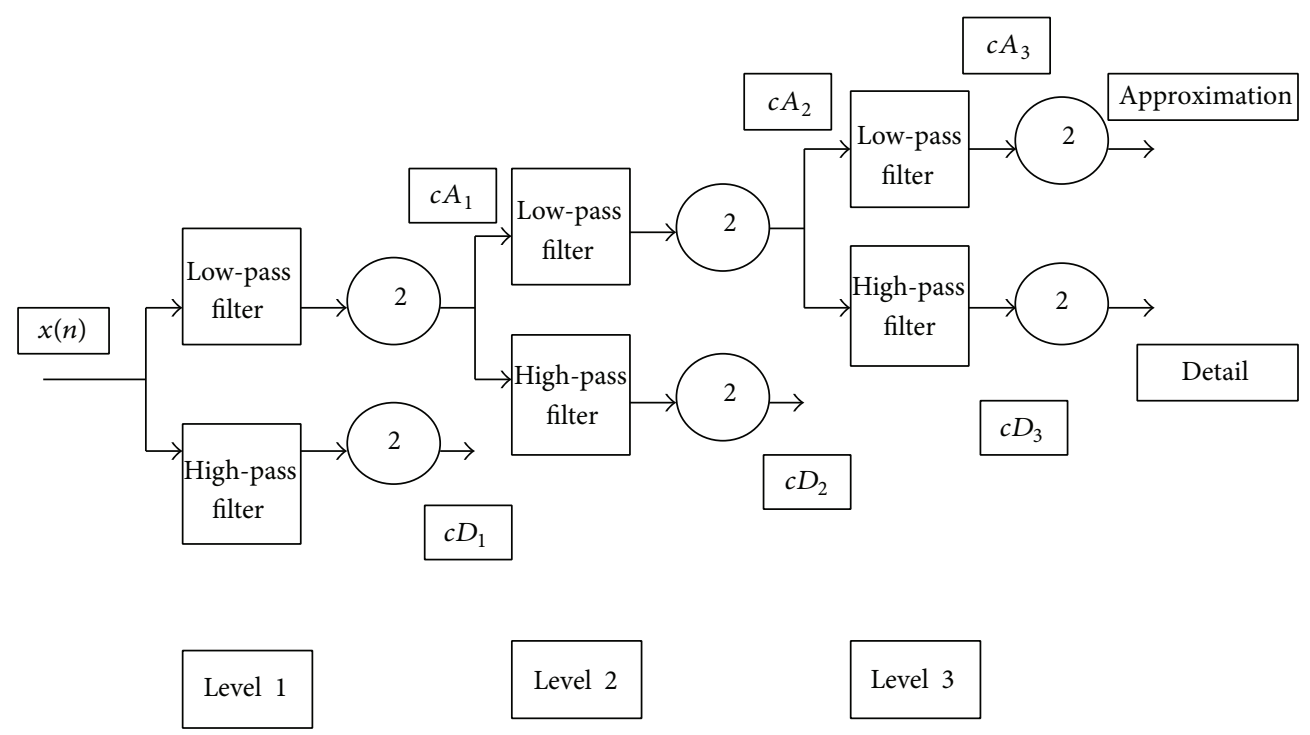

FIGURE 1: Diagram of multiresolution analysis of signal $x_{i}(t)$.

Such algorithm, which is amplification of discrete wavelet transform, is known as fast wavelet transform.

For analysis the following mother wavelet was used:

$$
\Psi_{m, n}(t)=2^{-m / 2} \Psi\left(2^{-m} t-n\right),
$$

where $n$ is coefficient of time translation and $m$ is coefficient of scale (compression).

In the first step threshold values for detail coefficients at every level of decomposition are determined according to the following relationship:

$$
\mathrm{THR}_{J}=\sqrt{2 \log \left\|c D_{j}\right\|}
$$

The next step is the modification of values of the $j$ th level detail coefficients basis of appointed threshold. This method is called soft thresholding procedure as follows:

$$
c D_{j}(t)=\left\{\begin{array}{l}
\operatorname{sgn}\left(c D_{j}(t)\right)\left(|x|-\mathrm{THR}_{j}\right) ; c D_{j}(t)>\mathrm{THR}_{j}, \\
0 ; c D_{j}(t) \leq \mathrm{THR}_{j} .
\end{array}\right.
$$

The final step of the analysis is reconstruction of signal $x_{i}(t)$ based of approximation coefficients chosen $i$ th level of decomposition $\left(c A_{i}\right)$ and modified detail coefficients from $i$ th $\left(c D_{i}\right)$ as well as higher levels of decomposition

$$
\begin{aligned}
x_{i}(t)= & \sum_{n} c A_{m, n} \Phi_{k}\left(2^{-m} t-n\right) \\
& +\sum_{m=m_{0}}^{m_{k}} \sum_{n} c D_{m, n} \Psi_{m, n}\left(2^{-m} t-n\right),
\end{aligned}
$$

where $\Phi_{k}(t)$ is scaling function from $k$ th level of decomposition and $\Psi_{m, n}(t)$ are wavelet functions for $m=m_{0}, \ldots, m_{k}$ levels of decomposition.

The advantages of wavelet methods are possibility of receiving good quality signal for beat to beat analysis and possibility of having high quality signal while averaging technique is impossible, as causing morphology distortion of electrocardiogram signals, it provides a way for analysing waveforms bounded in both frequency and duration, it allows signals to be stored more efficiently than by the Fourier transform, it can lead to better approximate real-world signals and it is well-suited for approximating data with sharp discontinuities. The disadvantage of wavelet method is that the wavelet transforms ignore polynomial components of the signal up to the approximation order of the basis.

2.2. Equation for Continuous Wavelet Transform. The wavelet transform equation is given by

$$
\operatorname{CWT}_{x}^{\Psi}(\tau, s)=\Psi(\tau, s)=\frac{1}{\sqrt{|s|_{t}}} \int x(t) \Psi^{*}\left(\frac{t-\tau}{s}\right) d t
$$

where $x(t)=$ given signal, $\tau=$ translation parameter, $s=$ scaling parameter $=1 / f$, and $\Psi(t)=$ mother wavelet.

2.3. Multiwavelet Method. Multiwavelets constitute a new chapter which has been added to wavelet theory in recent years. Recently, much interest has been generated in the study of the multiwavelets where more than one scaling functions and mother wavelet are used to represent a given signal. The first construction for polynomial multiwavelets was given by Albert, who used them as a basis for the representation of certain operators. Later, Geronimo, Hardin, and Massopust constructed a multiscaling function with 2 components using fractal interpolation.

In spite of many theoretical results on multiwavelet, their successful applications to various problems in signal processing are still limited. Unlike scalar wavelets in which Mallet's pyramid algorithm have provided a solution for good signal decomposition and reconstruction, a good framework for the application of the multiwavelet is still not available. Nevertheless, several researchers have proposed method of 
how to apply a given multiwavelet filter to signal and image decomposition.

2.4. Multiscaling Functions and Multiwavelets. The concept of multiresolution analysis can be extended from the scalar case to general dimension $r \mathrm{~N}$. A vector valued function $\varphi=\left[\varphi_{1} \varphi_{2}, \ldots, \varphi_{r}\right]^{T}$ belonging to $L^{2}(R)^{r}$ and $r \mathrm{~N}$ is called a multiscaling function if the sequence of closed spaces

$$
V_{j}=\operatorname{span}\left\{2^{j / 2} \varphi_{i}\left(2^{j}-k\right): 1 \leq i \leq r, k \in Z\right\} .
$$

$j \in Z$ constitute a multiresolution analysis (MRA) of multiplicity $r$ for $L^{2}(R)$. The multiscaling function must satisfy the two-scale dilation equation

$$
\varphi(t)=\sqrt{2} \sum_{k} G_{k} \varphi(2 t-k) .
$$

Now let $W_{j}$ denote a complementary space of $V_{j}$ in $V_{j+1}$. The vector valued function $\Psi=\left[\Psi_{1} \Psi_{2}, \ldots, \Psi_{r}\right]^{T}$ such that

$$
W_{j}=\operatorname{span}\left\{2^{j / 2} \varphi_{i}\left(2^{j}-k\right): 1 \leq i \leq r, k \in Z\right\} .
$$

$j \in Z$ is called a multiwavelet. The multiscaling function must satisfy the two-scale equation

$$
\Psi(t)=\sqrt{2} \sum_{k} H_{k} \varphi(2 t-k) .
$$

$H_{k} \in L^{2}(Z)^{r \times r}$ is an $r \times r$ matrix of coefficients. The twoscale equations (2) and (4) can be realized as a multifilter bank operating on $r$ input data streams and filtering them in two $2 r$ output data streams, each of which is downsampled by a factor of two. If $x(t)$ is the given signal and it is assumed that $x(t) \in V_{0}$, then

$$
x(t)=\sqrt{2} \sum_{k} V_{0, k}^{T} \varphi(t-k) .
$$

And the scaling coefficient $V_{1, k}^{T}$ of the first level can be considered as a result of low-pass multifiltering and downsampling as follows:

$$
V_{1, k}=\sum_{m} G_{m-2 k} V_{0, m} .
$$

Analogously, the first level multiwavelet coefficients $W_{1, k}$ are obtained using high-pass multifiltering and downsampling as follows:

$$
W_{1, k}=\sum_{m} H_{m-2 k} V_{0, m} .
$$

Full multiwavelet decomposition of the signal $x(t)$ can be found by iterative filtering of the scaling coefficient as follows:

$$
\begin{aligned}
V_{j, k} & =\sum_{m} G_{m-2 k} V_{j-1, m}, \\
W_{j, k} & =\sum_{m} H_{m-2 k} V_{j-1, m} .
\end{aligned}
$$

Note that $V_{j, k}$ and $W_{j, k}$ are $r \times 1$ column vectors.

\subsection{Advantages of Wavelets and Multiwavelets Compared to Conventional Filtering Techniques}

(i) The Fourier transform fails to analyze the nonstationary signal, whereas wavelet transform allows the components of a nonstationary signal to be analyzed.

(ii) Wavelet transform holds the property of multiresolution to give both time and frequency domain information in a simultaneous manner.

(iii) A set of wavelets which are complementary can decompose the given data without gaps or overlap so that the decomposition process becomes mathematically reversible.

2.6. Comparison between Wavelet and Multiwavelet. Multiwavelets contain multiple scaling functions, whereas scalar wavelets contain one scaling function and one wavelet. This leads to more degrees of freedom in constructing wavelets. Therefore, opposed to scalar wavelets, properties such as compact support, orthogonality, symmetry, vanishing moments, and short support can be gathered simultaneously in multiwavelets which are fundamental in signal processing.

The increase in degree of freedom in multiwavelets is obtained at the expense of replacing scalars with matrices, scalar functions with vector functions, and single matrices with block of matrices. However, prefiltering is an essential task which should be performed for any use of multiwavelet in signal processing.

\section{Results and Discussions}

\subsection{Data Collection}

3.1.1. MIT-BIH Arrhythmia Database. MIT-BIH arrhythmia database consists of 48-half-hour electrocardiogram recordings. The recordings were digitized at $360 \mathrm{~Hz}$ (samples per second per channel) with 11-bit resolution over $10 \mathrm{mV}$. The simulations were carried out in MATLAB environment R2010b. Various benchmark records from the MIT-BIH database were considered for this study.

\section{(A) Performance Analysis of Wavelet Based Denoising Method} for Electrocardiogram

Wavelet Denoising Using Biorthogonal 1D Wavelet. Figure 2 shows the wavelet denoising for the record $103 \mathrm{~m}$ in which level 1 approximation coefficient $d_{1}$ for Biorthogonal wavelet shows that there is maximum noise in it. Hence reconstruction of the original signal to obtain the denoised electrocardiogram from $d_{1}$ coefficients will also contain the maximum noise. The level 4 decomposition contains the least noise and hence reconstruction is done using $d_{4}$.

Original and Denoised Signals Using Biorthogonal Wavelet. Figure 3 shows the comparison of the original signal and the denoised signal using Biorthogonal wavelet at level 4 decomposition for the record $103 \mathrm{~m}$. In this the signal to noise ratio obtained is $32.2094 \mathrm{~dB}$ and the power line noise 


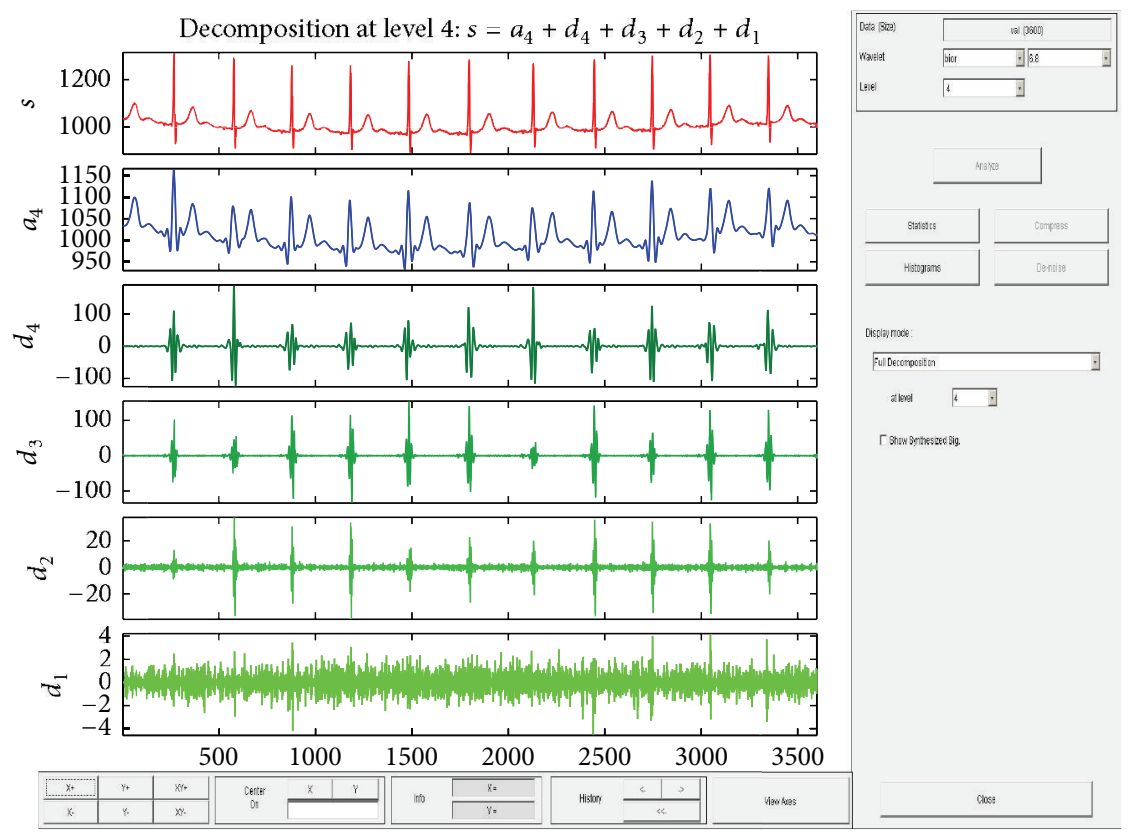

FIGURE 2: Wavelet denoising for ECG record no $103 \mathrm{~m}$ using Biorthogonal 1D wavelet.



FIGURE 3: Original and denoised signals for ECG record no $103 \mathrm{~m}$ using Biorthogonal wavelet.

is reduced for this record. Figure 4 shows the approximation and coefficient details after the signal in record $114 \mathrm{~m}$ has been subjected to the Biorthogonal wavelet transform and Figure 5 shows the original and denoised electrocardiogram in the record $114 \mathrm{~m}$. The signal to noise ratio obtained is $9.184 \mathrm{~dB}$. This shows that the noise has to be removed further to enhance the quality of the obtained electrocardiogram.

\section{(B) Performance Analysis of Multiwavelet Based Denoising Method for Electrocardiogram}

Biothogonal Based Multiwavelet Denoising. The multiwavelet denoising for the record $103 \mathrm{~m}$ shown in Figure 6 yields a signal to noise ratio of $35.5220 \mathrm{~dB}$ in which the power line noise gets removed. Figure 7 shows the multiwavelet denoising for the record $114 \mathrm{~m}$ and the signal to noise ratio obtained is $13.4022 \mathrm{~dB}$ because of the removalof power line noise.

3.2. Performance Comparison of Wavelet and Multiwavelet Methods. Comparison of signal to noise ratio for wavelet and multiwavelet based denoising techniques for various electrocardiogram records.

\section{Conclusion}

The inference from Tables 1,2,3, and 4 is that the output signal to noise ratio value of multiwavelet denoising functions is 


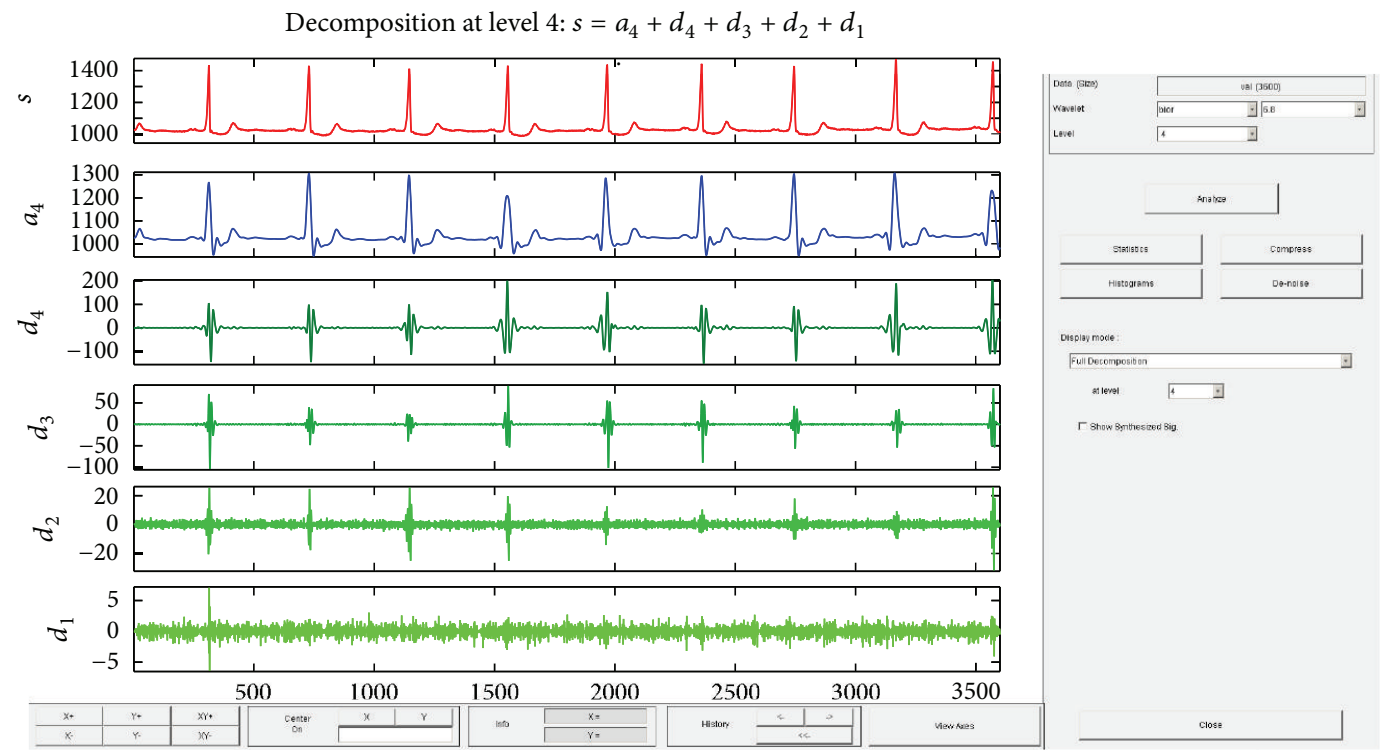

Figure 4: Wavelet denoising for ECG record no $114 \mathrm{~m}$ using Biorthogonal 1D wavelet.

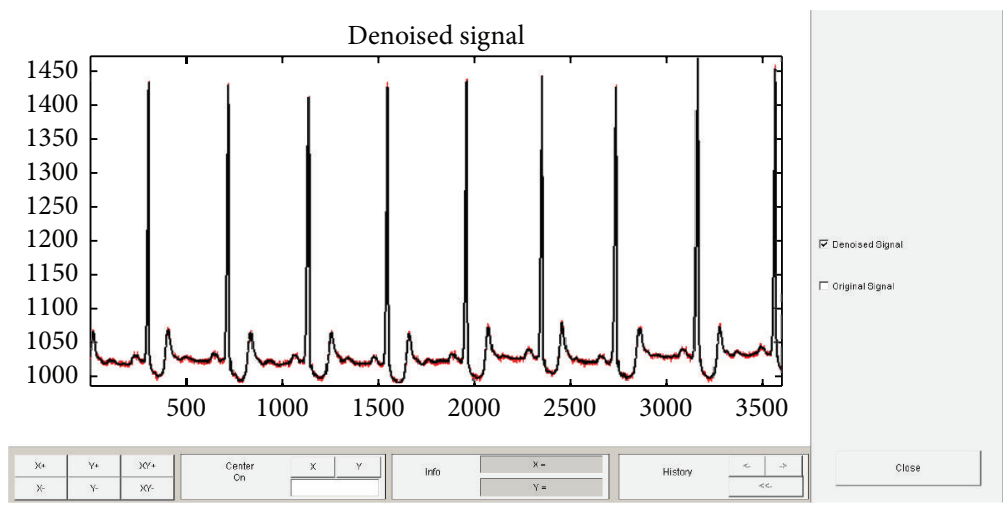

— Original ECG signal
— Denoised ECG signal

FIGURE 5: Original and denoised signals for ECG record no $114 \mathrm{~m}$ using Biorthogonal wavelet.

TABle 1: Performance comparison of wavelet and multiwavelet for record $100 \mathrm{~m}$.

\begin{tabular}{lcc}
\hline \multirow{2}{*}{ Wavelet family } & \multicolumn{2}{c}{ SNR (dB) } \\
& Wecord no 100 m \\
\hline Bio 6.8 & 11.5708 & Multiwavelet \\
Db 3 & 2.7306 & 29.8328 \\
Db10 & 0.561 & 21.7829 \\
Coif 4 & 2.466 & 7.3489 \\
Sym 4 & 9.0630 & 3.6112 \\
\hline
\end{tabular}

greater than the signal to noise value of wavelet functions. The table also indicates that the Daubechies wavelet 10 has better denoising capability than when compared to corresponding values of the wavelet denoising as the shape of this wavelet
TABle 2: Performance comparison of wavelet and multiwavelet for record $103 \mathrm{~m}$.

\begin{tabular}{lcc}
\hline \multirow{2}{*}{ Wavelet family } & \multicolumn{2}{c}{ SNR (dB) } \\
& Record no 103 m \\
\hline Bio 6.8 & 32.2094 & Multiwavelet \\
Db 3 & 15.827 & 35.5220 \\
Db10 & 19.1099 & 27.7849 \\
Coif 4 & 24.1340 & 33.1183 \\
Sym 4 & 24.6220 & 29.7007 \\
\hline
\end{tabular}

is more close to the shape of electrocardiogram. The increase in signal to noise ratio value indicates that there is no loss in the information contained in the original electrocardiogram signal and multiwavelet has better denoising capability to 




FIGURE 6: Multiwavelet denoising for ECG record no $103 \mathrm{~m}$ using Biorthogonal 1D wavelet.

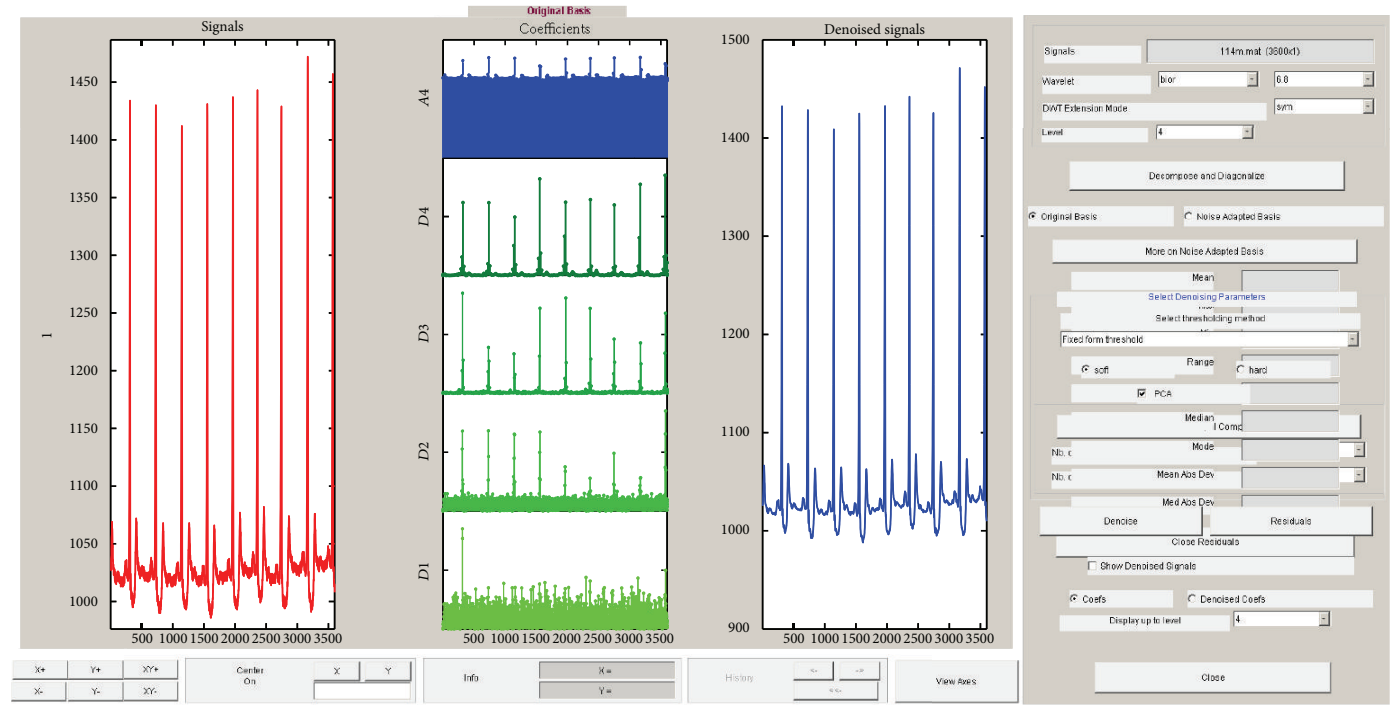

FIgURE 7: Multiwavelet denoising for ECG record no $114 \mathrm{~m}$ using Biorthogonal 1D wavelet.

TABLE 3: Performance comparison of wavelet and multiwavelet for record $114 \mathrm{~m}$.

\begin{tabular}{lcc}
\hline Wavelet family & \multicolumn{2}{c}{ SNR (dB) } \\
& Record no 114 m \\
\hline Bio 6.8 & 9.184 & Multiwavelet \\
Db 3 & 28.6913 & 13.4022 \\
Db10 & 18.2315 & 31.0045 \\
Coif 4 & 19.3813 & 28.5193 \\
Sym 4 & 29.6574 & 36.6501 \\
\hline
\end{tabular}

TABLE 4: Performance comparison of wavelet and multiwavelet for record $201 \mathrm{~m}$.

\begin{tabular}{lcc}
\hline Wavelet family & \multicolumn{2}{c}{ SNR (dB) } \\
& Record no 201 m \\
\hline Bio 6.8 & 11.5636 & Multiwavelet \\
Db 3 & 20.1344 & 32.3220 \\
Db10 & 21.2696 & 29.3001 \\
Coif 4 & 17.8031 & 33.7040 \\
Sym 4 & 24.0735 & 21.2874 \\
\hline
\end{tabular}


remove the power line noise in the various electrocardiogram records.

\section{Conflict of Interests}

The authors declare that they have no conflict of interests regarding the publication of this paper.

\section{References}

[1] C. Germaine, F. Halberg, T. Breus et al., "Non-photic solar associations of heart rate variability and myocardial infarction," Journal of Atmospheric and Solar-Terrestrial Physics, vol. 64, no. 5, pp. 707-720, 2002.

[2] M. M. Goudarzi, A. Taheri, M. Pooyan, and R. Mahboobi, "Multiwavelet and biological signal processing," International Journal of Information Technology, vol. 24, pp. 264-272, 2006.

[3] M. Kania, M. Fereniec, and R. Maniewski, "Wavelet denoising for multi-lead high resolution ECG signals," Measurement Science Review, vol. 7, Section 2, no. 4, pp. 30-33, 2007.

[4] K. Helenprabha and A. M. Natarajan, "FPGA implementation of gamma filter for extracting fetal electrocardiogram," IETE Journal of Research, no. 53, pp. 433-440, 2007.

[5] F. C. Chang, C. K. Chang, K. Y. Chi, and Y. D. Lin, "Evaluation measures for adaptive PLI filters in ECG signal processing," Computers in Cardiology, vol. 34, pp. 529-532, 2007.

[6] M. Alfaouri and K. Daqrouq, "ECG Signal Denoising by wavelet transform thresholding," The American Journal of Applied Sciences, vol. 53, pp. 276-281, 2008.

[7] Z. Zhidong and M. Chan, "A novel cancellation method of powerline interference in ECG signal based on EMD and adaptive filter," in Proceedings of the 11th IEEE International Conference on Communication Technology, pp. 517-520, 2008.

[8] M. Kaur and B. Singh, "Powerline interference reduction in ECG using combination of MA method and IIR notch," International Journal of Recent Trends in Engineering, vol. 2, no. 6, pp. 125-129, 2009.

[9] A. K. M. F. Haque, M. H. Ali, M. A. Kiber, and M. T. Hasan, "Detection of small variations of ECG features using wavelet," ARPN Journal of Engineering and Applied Sciences, vol. 4, no. 6, pp. 27-30, 2009.

[10] Y.-F. Tan and D. Lei, "Study on Wavelet Transform in the processing for ECG signals," in Proceedings of the WRI World Congress on Software Engineering (WCSE '09), vol. 9, pp. 515518, IEEE Computer Society, May 2009. 


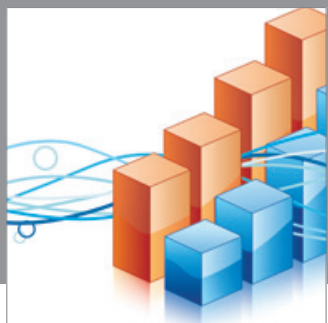

Advances in

Operations Research

mansans

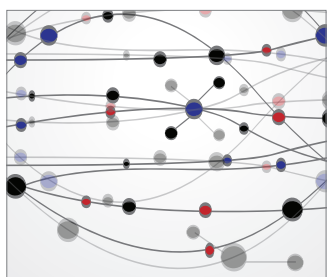

The Scientific World Journal
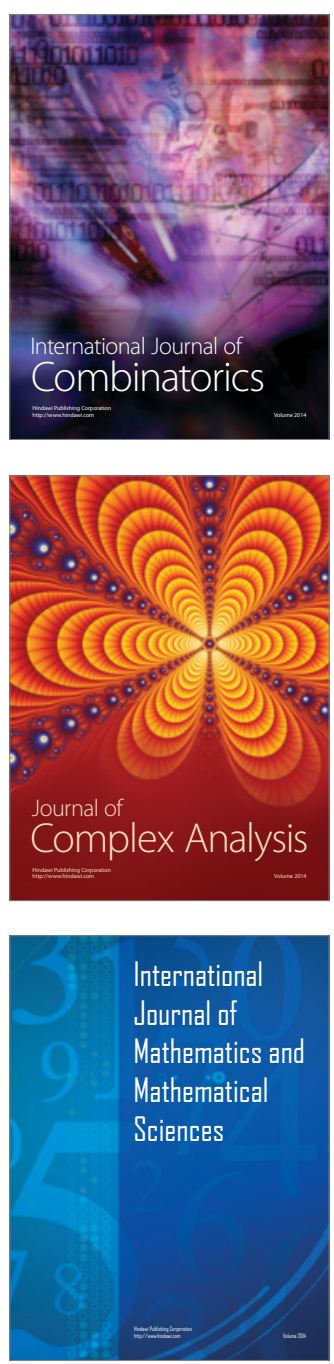
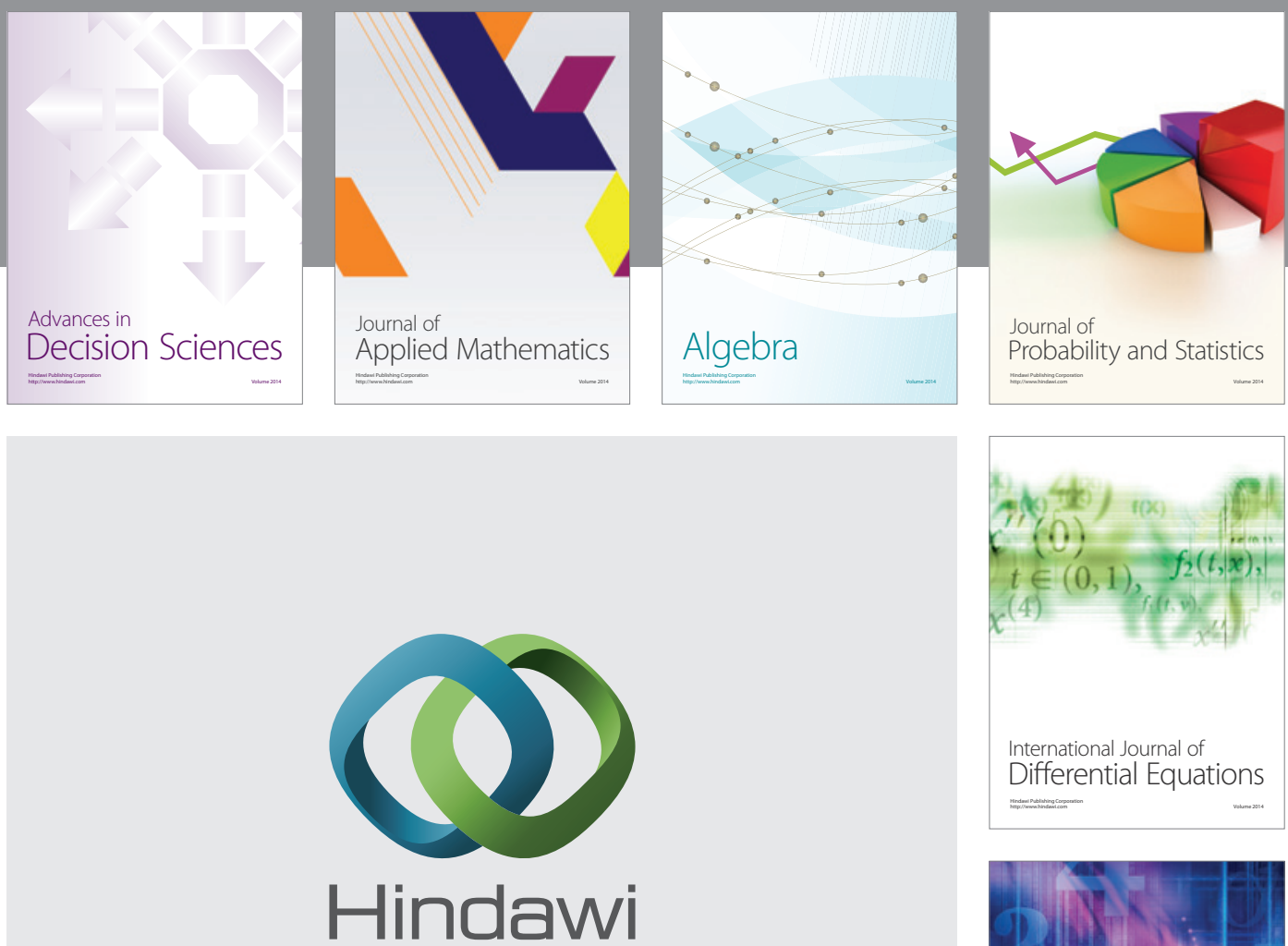

Submit your manuscripts at http://www.hindawi.com
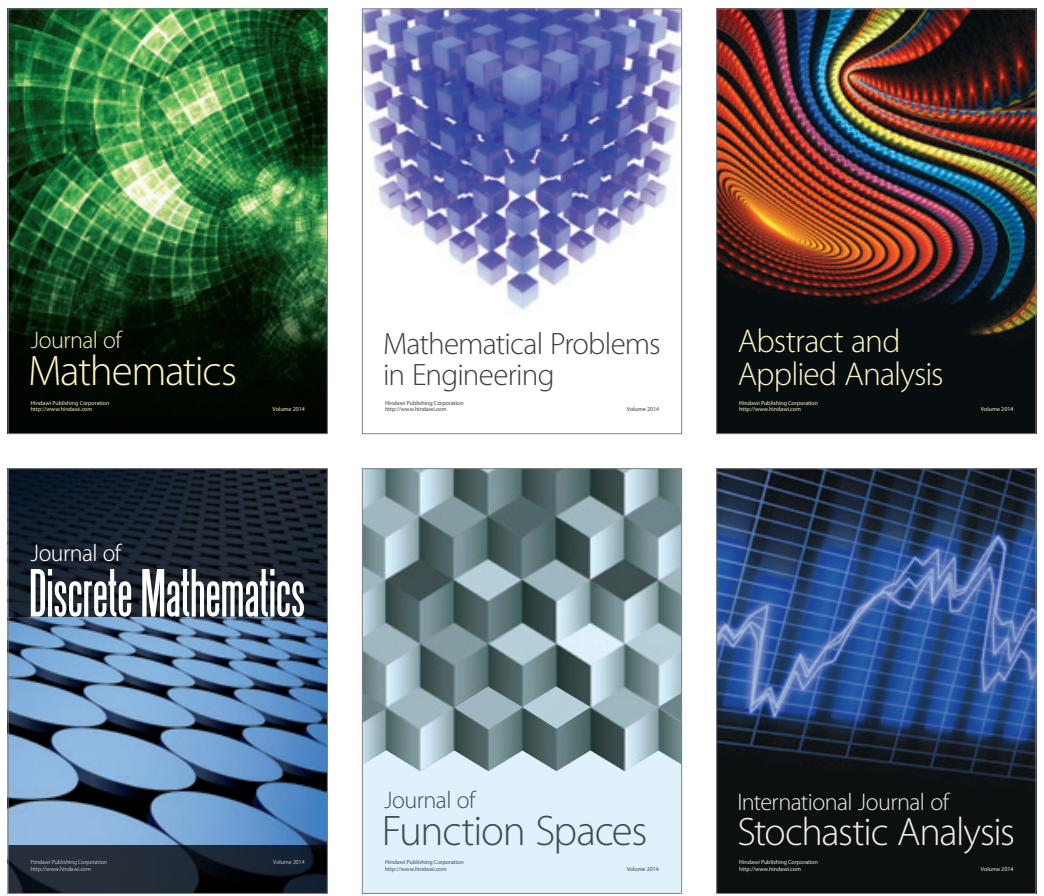

Journal of

Function Spaces

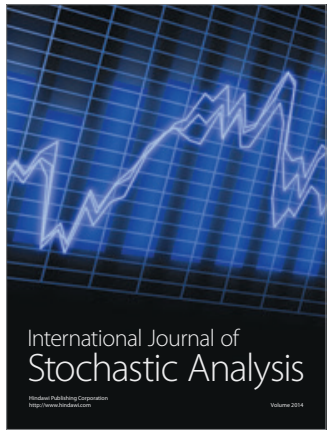

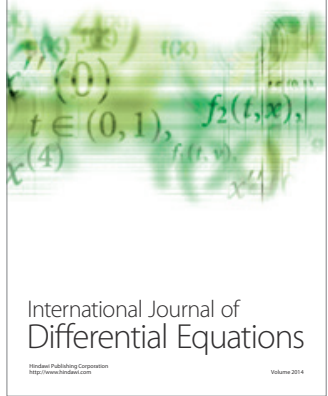
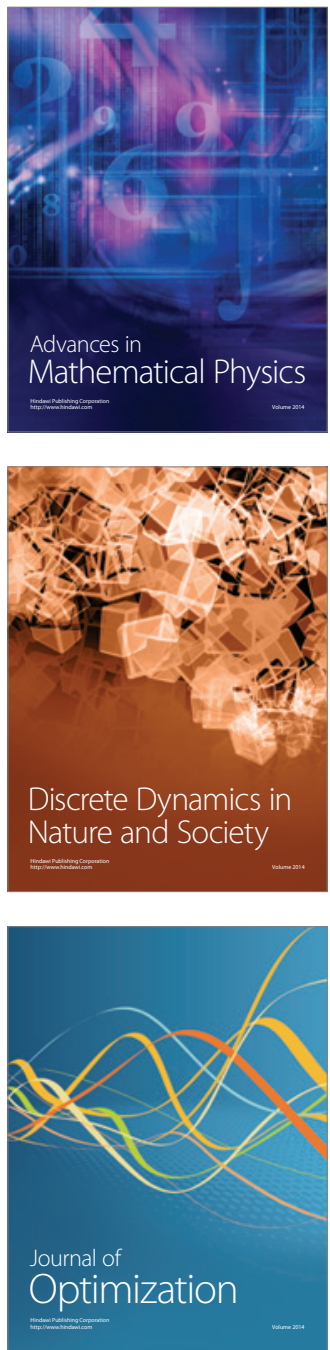